\title{
Air-to-ground Attack Path Planning Method without Terrain Restriction
}

\author{
Xiaowen Duan \\ School of Electronic and Information \\ Northwestern Polytechnical University \\ Xi'an, China \\ xwduan2008@163.com
}

\author{
Xiaoguang Gao \\ School of Electronic and Information \\ Northwestern Polytechnical University \\ Xi'an, China \\ cxg2012@nwpu.edu.cn
}

\begin{abstract}
Through the analysis of the research status, battle features and operational requirements on air-to-ground attack, the paper proposes a new air-to-ground attack path planning method. Firstly, the characteristic point calculation method is put forward based on the scheduled attack direction and weapon performance, then the trajectory point solving method based on characteristic point is put forward, subsequently proposes the time estimation method of combat units reaching trajectory point and muti-formation time cooperation method, finally the simulation result shows the effectiveness of the method.
\end{abstract}

Keywords-path planning; air-to-ground attack; time cooperation; great circle

\section{INTRODUCTION}

In modern war, using strike aircraft to attack ground target, can not only eleminate the effective strength of the enemy to obtain air domination, but also adjust oil load and bomb load whenever to implement flexible attack, therefore using aviation weapon to implement air-toground attack has become the important pillar when realizing strategic goal[1-3]. Path planning refers to find the optimal trajectory from the starting point to the ending point of moving object under specific constraint[4-7]. At present, study on air-to-ground attack is almost based on the assumption that aircraft flies under terrain restriction[810].

But actually, in many cases there is no terrain restrictions when acting air-to-ground attack, so it's not necessary to consider terrain restriction in this case. Based on this, the paper proposes a new air-to-ground attack path planning method, through which could quickly get the flying path of attack formation by taking into consideration the aircraft's combat performance and the ground threat's characteristic parameters, and meanwhile supports the time cooperation of muti-formation.

\section{PATH PLANNING OF AIR-TO-GROUND ATTACK}

In this section, firstly the relative coordinate system and their relationship is introduced, then begins the research on path planning method of air-to-ground strike formation.

In order to describe easier, here uses " $\mathrm{Rg}$, " $\mathrm{R}_{\lambda-\phi}$ " and " $\mathrm{R}_{\mathrm{o}}$ " to stand for the local coordinate system, space geodetic coordinate system and space rectangular coordinate system respectively. The base coordinate system (" $\mathrm{R}_{\mathrm{a}}$ ") can be obtained through rotating $\mathrm{X}$ axis of "Rg" by $\theta_{\mathrm{A}}$.

The relationship between the above coordinate system can be expressed as follows:

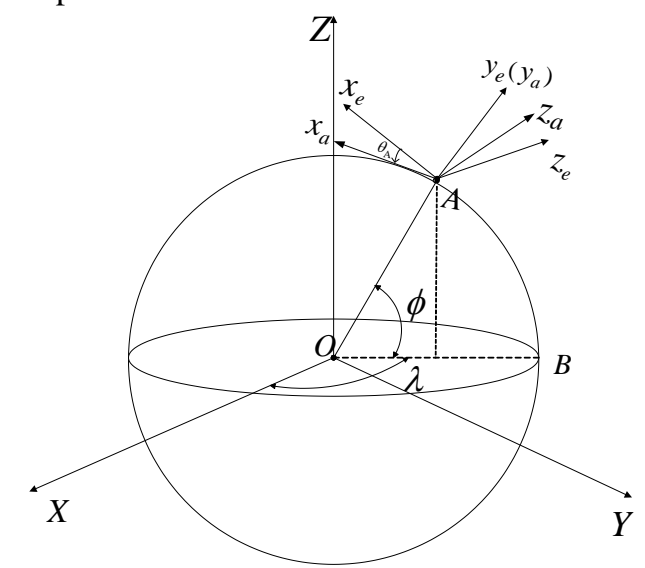

Figure 1. relationship between the coordinate systems

Supposing $A_{E}^{L}$ represents the transfer matrix from " $\mathrm{R}_{\lambda-\phi} "$ to $" \mathrm{Rg}^{\prime}, A_{L}^{A}$ represents the transfer matrix from "Rg" to "Ra", $A_{E}^{A}$ represents the transfer matrix from " $\mathrm{R}_{\lambda-\phi}$ " to " $\mathrm{R}_{\mathrm{a}}$, , then $A_{E}^{L}, A_{L}^{A}$ and $A_{E}^{A}$ can be obtained from (1) to (3):

$$
\begin{gathered}
A_{E}^{L}=\left[\begin{array}{ccc}
-\sin \phi \cos \lambda & -\sin \phi \sin \lambda & \cos \phi \\
\cos \phi \cos \lambda & \cos \phi \sin \lambda & \sin \phi \\
-\sin \lambda & \cos \lambda & 0
\end{array}\right] \\
A_{L}^{A}=\left[\begin{array}{ccc}
\cos \theta_{A} & 0 & -\sin \theta_{A} \\
0 & 1 & 0 \\
\sin \theta_{A} & 0 & \cos \theta_{A}
\end{array}\right] \\
A_{E}^{A}=A_{L}^{A} A_{E}^{L}
\end{gathered}
$$




\section{A. Characteristic Point Calculation}

The typical air-to-ground attack formation is consisted of main attacker, associated attacker, associated electronicjamming aircraft and escort aircraft, and in the planning path they keeps the fixed team formation. So the attack path of the attack formation can be determined by the path of the main attacker. In other words, the calculation of attack formation path can be simplified as the calculation of main attacker path.

Supposing the side view of strike path is as follows:

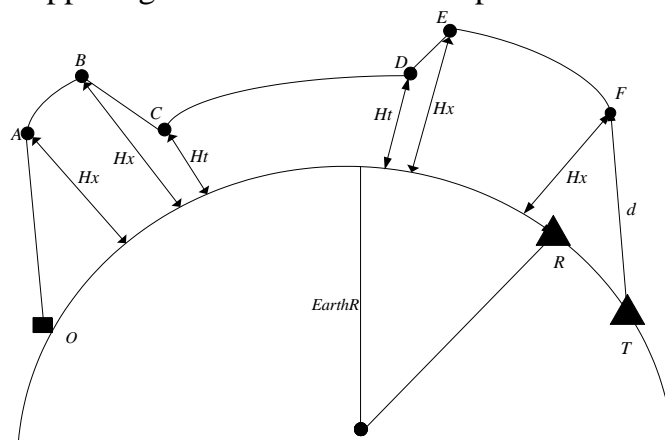

Figure 2. Side view of strike path

In Fig.2 the whole path of strike formation is in the plane determined by takeoff base and scheduled release point, so in " $\mathrm{R}_{\mathrm{a}}$ " coordinate system, the $Z$ component of $A 、 B 、 C 、 D 、 E$ and $F$ is all equal to $0 . A \rightarrow$ $B \rightarrow C \rightarrow D \rightarrow E \rightarrow F$ is the flying path of strike formation( $A$ : the coordinate from base $O$ to the scheduled cruise altitude; $B$ : the coordinate of the cruise emergence point; $C$ : the coordinate from cruise emergency point to scheduled penetration altitude; $D:$ the coordinate of penetration emergency point; $E$ : the point finished climb; $F$ : the scheduled release point).

Next, the paper will give the computational method of trajectory point under " $\mathrm{R}_{\mathrm{a}}$ " confirmed by the target.

Before computing the coordinate of trajectory points, the scheduled bomb-release direction should be fixed.

Supposing the scheduled bomb-release direction is $\theta_{A}(R a d)$, cruise altitude is $H x(m)$, penetration altitude is $H t(m)$, range of ASM is $d_{s}(m)$, the equivalent radius is $R(m)$, then

step to calculating the coordinate of $F$ :

Supposing the coordinate of $F$ is $\left(x_{F G}, y_{F G}, z_{F G}\right)^{T}$ in " $\mathrm{Rg} "$ confirmed by the target and $\left(x_{F A}, y_{F A}, 0\right)^{T}$ in " $\mathrm{R}_{\mathrm{a}}$ " confirmed by base $O$, it's coordinate in " $\mathrm{R}_{\lambda-\phi}$ " is $\left(\lambda_{F}, \varphi_{F}, h_{F}\right),\left(x_{F G 0}, y_{F G 0}, 0\right)^{T}$ stands for the coordinate of $F$ in " $\operatorname{Rg} "$ when $\theta_{A}=0$, then

$$
\left\{\begin{array}{l}
x^{2}+(y+R)^{2}=(R+h)^{2} \\
(x-a)^{2}+(y-b)^{2}=(d-c)^{2}
\end{array}\right.
$$

Here $a=b=c=0, d=d_{s}, h=H x$.
After solving (4) $\left(x_{F G 1}, y_{F G 1}, 0\right)^{T}$ and $\left(x_{F G 2}, y_{F G 2}, 0\right)^{T}$ is obtained, $\left(x_{F G 0}, y_{F G 0}, 0\right)^{T}$ takes the one whose $\mathrm{X}$ component is positive. And

$$
(x, y, z)^{T}=A_{L}^{A}\left(x_{F G 0}, y_{F G 0}, 0\right)^{T}
$$

Here $A_{L}^{A}$ stands for the transfer matrix from " $\mathrm{Rg}$ " to " $\mathrm{R}_{\mathrm{a}}$. Calculate the corresponding coordinate of $(x, y, z)^{T}$ as $\left(\lambda_{F}, \varphi_{F}, h_{F}\right)$, then get the corresponding coordinate as $\left(x_{A F}^{\prime}, y_{A F}^{\prime}, z_{A F}^{\prime}\right)^{T}$ in " $\mathrm{Rg} "$ confirmed by base $O$, finally $\left(x_{F A}, y_{F A}, 0\right)^{T}$ is obtained by converting $\left(x_{A F}^{\prime}, y_{A F}^{\prime}, z_{A F}^{\prime}\right)^{T}$ to " $\mathrm{R}_{\mathrm{a}}$ " confirmed by base $O$.

step to calculating the coordinate of $B$ and $D$ :

The max detection distance $d_{b}$ of warning radar to target with altitude $H x$ can be calculated from[12], Supposing the coordinate of $B$ and warning radar is $\left(x_{B A}, y_{B A}, 0\right)^{T}$ and $(a, b, c)^{T}$ in " $\mathrm{R}_{\mathrm{a}}$ " confirmed by base $O$, taking $d=d_{b}, h=H x$ and $(a, b, c)^{T}$ into (4) gets $\left(x_{B 1}, y_{B 1}, 0\right)^{T}$ and $\left(x_{B 2}, y_{B 2}, 0\right)^{T},\left(x_{B A}, y_{B A}, 0\right)^{T}$ takes the one whose module is smaller. The corresponding $\left(x_{B A}, y_{B A}, 0\right)^{T}$ for other warning radar can be obtained in the same way, the desired value is the one whose module is minimum.

Supposing $d_{d}$ is the max detection distance of warning radar to target with altitude $H t$, the step to calculate the coordinate of $D$ and $B$ is the same but replace $d=d_{d}$ and $h=H t$.

step to calculating the coordinate of $C$ and $E$ :

Supposing the coordinate of $B$ and $C$ is $\left(x_{B A}, y_{B A}, 0\right)^{T}$ and $\left(x_{C A}, y_{C A}, 0\right)^{T}$ in " $\mathrm{R}_{\mathrm{a}}$ " confirmed by base $O$, the distance from $B$ to $C$ is $d_{b c}$, then

$$
\left\{\begin{array}{l}
a=x_{B A} \\
b=y_{B A} \\
c=0 \\
h=H t \\
d=d_{b c} \\
d_{b c}=\sqrt{m^{2}+n^{2}} \\
m=H x-H t \\
n=m * \sqrt{V^{2}-V_{\perp}^{2}} / V_{\perp}
\end{array}\right.
$$

Taking (6) into (4) gets $\left(x_{C A 1}, y_{C A 1}, 0\right)^{T}$ and $\left(x_{C A 2}, y_{C A 2}, 0\right)^{T},\left(x_{C A}, y_{C A}, 0\right)^{T}$ takes the one whose module 
is larger. $V$ and $V_{\perp}$ means the speed and max climb (dive) speed respectively.

Set $d_{d e}$ as the distance from $D$ to $E$, then

$$
\left\{\begin{array}{l}
a=x_{D A} \\
b=y_{D A} \\
c=0 \\
h=H x \\
d=d_{d e}
\end{array}\right.
$$

Taking (7) into (4) gets $\left(x_{E A 1}, y_{E A 1}, 0\right)^{T}$ and $\left(x_{E A 2}, y_{E A 2}, 0\right)^{T},\left(x_{E A}, y_{E A}, 0\right)^{T}$ takes the one whose module is larger.

step to calculating the coordinate of $A$ :

The coordinate of $A$ in " $\mathrm{R}_{\mathrm{a}}$ " confirmed by base $O$ is $\left(H x * \sqrt{V^{2}-V_{\perp}^{2}} / V_{\perp}, H x, 0\right)^{T}$.

\section{B. Trajectory Point Solving Method}

Fig. 3 gives the side view of air-to-ground formation strike path:

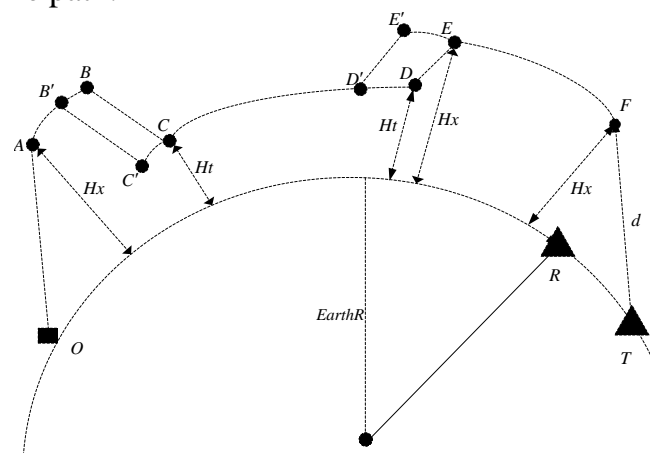

Figure 3. Side view of strike path

$$
\text { Taking } C=\left(O A, A B^{\prime}, B^{\prime} C^{\prime}, C^{\prime} D^{\prime}, D^{\prime} E^{\prime}, E^{\prime} F\right) \text { to depict }
$$

the 6 stages of the whole strike process, $V_{i}$ means the speed in stage $i$, in order to strengthen the suddenness of strike, it's better for attack formation to consume less time, the aim can be expressed as follows:

$$
\min 0=\sum_{i=1}^{6} \boldsymbol{C}_{i} / V_{i}
$$

Meanwhile, in order to make sure the attack formation has stronger battlefield adaptive ability, it's better for attack formation has more surplus oil, the aim can be expressed as follows:

$$
\max 0=m_{\text {left }}
$$

Where $m_{\text {left }}$ means the surplus oil for attacker after finished flying, $m_{c a}, m_{c x}$ and $m_{c t}$ means the oil consumption rate for attacker at the climb, cruise and penetration stage respectively, then

$$
m_{\text {left }}=m-\sum_{i=1,3,5} \boldsymbol{C}_{i} / m_{c a}-\sum_{i=2,6} \boldsymbol{C}_{i} / m_{c x}-\boldsymbol{C}_{4} / m_{c t}
$$

The objective function for trajectory point solving is constructed as follows:

$\min =\alpha_{1} \min 0+\alpha_{2} / \max 0$

Where $\alpha_{1}>0$ and $\alpha_{2}>0$, also $\alpha_{1}+\alpha_{2}=1$.

The constraint condition is:

$$
\left\{\begin{array}{l}
\alpha_{3} B \cdot x \leq B^{\prime} . x \leq B \cdot x \\
\alpha_{4} D \cdot x \leq D^{\prime} \cdot x \leq D \cdot x \\
m_{\text {left }} \geq m_{\min }
\end{array}\right.
$$

Where $\eta \leq \alpha_{3}, \alpha_{4} \leq 1, \eta$ may take the minimum of all the $X$ component of $D^{\prime}$. In (12) B.x means the $X$ component of $B$.

\section{MUTI-FORMATION TIME COOPERATION PLANNING}

The focus of muti-formation time cooperation planning is the takeoff time cooperation planning, it's aim is to make sure multiple formation arrive the scheduled place synchronously and strengthen the suddenness of strike, so that the enemy don't have enough time to make plan for defense and then the strike effect is improved. According to the proposed strike path, the muti-formation time cooperation planning is to coordinate the takeoff time to make sure they arrive $D^{\prime}$ at the same time.

According to the scheduled fly path, the arrival time of any single formation to any trajectory point can be estimated approximately. If the strike formation keeps the constant speed $V$ in the whole stage, the arrival time for point $i$ is $t_{i}=\sum_{i=1}^{6} D_{i} / V$, instead if the speed differs in each stage, the arrival time for point $i$ is $t_{i}=\sum_{i=1}^{6} D_{i} / V_{i}$

The step to fulfill muti-formation time cooperation is: firstly assume that the arrival time of formation $i$ to point $D^{\prime}$ is $t_{i}$, set $\max \left(t_{i}, i=1,2 \ldots n\right)$ as the maximum of the time to $D^{\prime}$ for the whole formation ,and then the takeoff time of formation $i$ can be obtained as $\max \left(t_{i}, i=1,2 \ldots n\right)-t_{i}$.

\section{EXAMPLE}

Now carries on the simulation of scenario Table 1-3 based on the proposed path planning method, where Table

\begin{tabular}{|c|c|c|}
\hline Unit Position & Longitude $\left(^{\circ}\right)$ & Latitude $\left(^{\circ}\right)$ \\
\hline Base 1 & 116.930 & 28.192 \\
\hline Base 2 & 114.826 & 25.306 \\
\hline
\end{tabular}
1 gives the position information of target and it's ambient warning radar, Table 2 gives the main performance parameter of warning radar, Table 3 gives the related parameter of the two strike formation.

TABLE I. POSITION OF TARGET AND RADARS 


\begin{tabular}{|c|c|c|}
\hline Target & 118.5 & 22 \\
\hline Radar 1 & 118.5 & 22.630 \\
\hline Radar 2 & 117.910 & 22.313 \\
\hline Radar 3 & 119.089 & 22.314 \\
\hline Radar 4 & 117.913 & 21.684 \\
\hline Radar 5 & 119.087 & 21.684 \\
\hline Radar 6 & 118.5 & 21.370 \\
\hline
\end{tabular}

TABLE II. PERFORMANCE PARAMETER OF RADAR

\begin{tabular}{|c|c|}
\hline Performance parameter & Value \\
\hline Transmit power $(K w)$ & 3 \\
\hline Main lobe gain $(d B)$ & 30 \\
\hline Receiver bandwidth $(K H Z)$ & 10 \\
\hline Operating frequency $(M H Z)$ & 3000 \\
\hline Half-power beam width $\left(^{\circ}\right)$ & 5 \\
\hline Noise power $(w)$ & $5 \mathrm{e}-17$ \\
\hline polarization coefficient & 0.5 \\
\hline Pulse accumulation & 8 \\
\hline
\end{tabular}

TABLE III. PARAMETERS OF ATTACK FORMATION

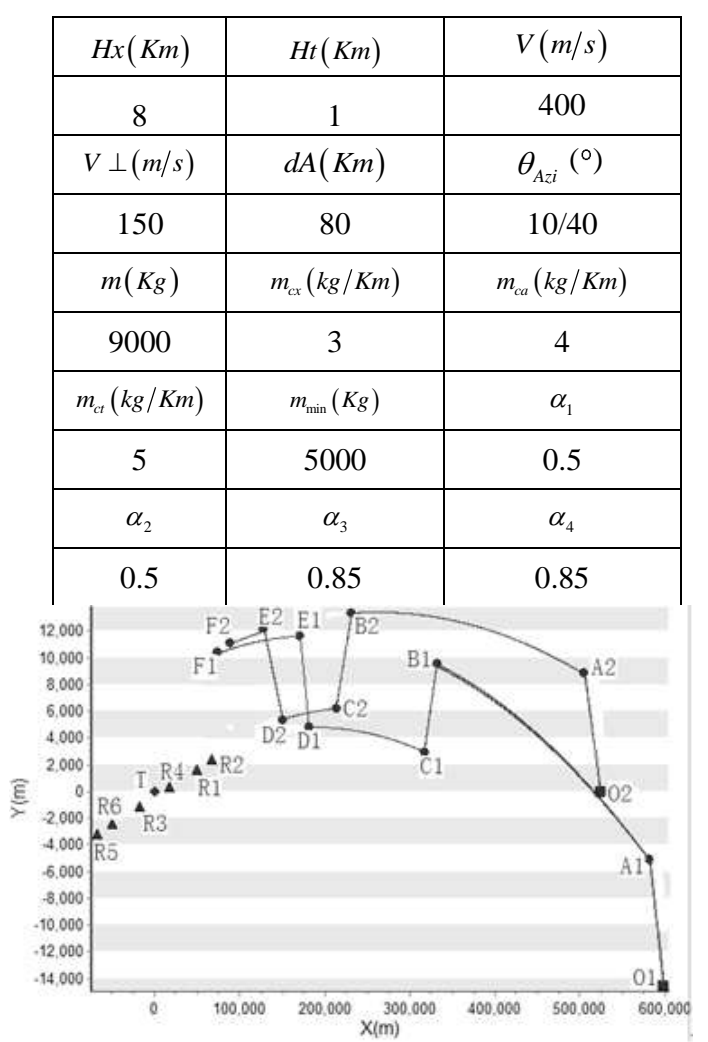

Figure 4. Side view of planning path

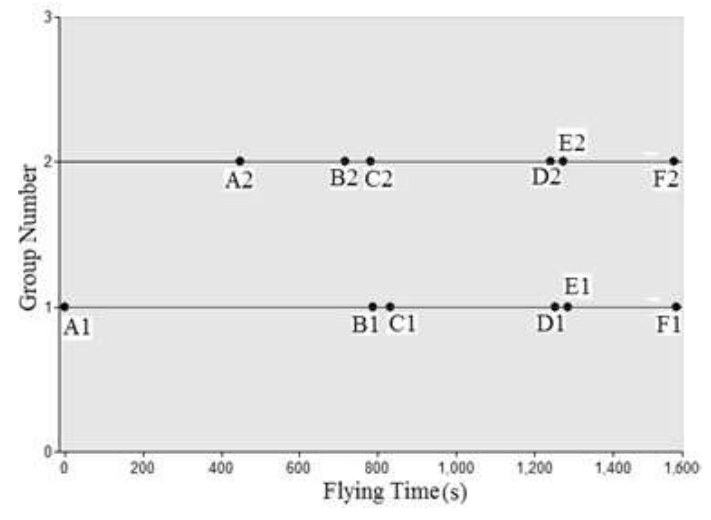

Figure 5. Time profile of strike formation

Fig.4 gives the two formation trajectory under the target-determined " $\mathrm{Rg}$ ", it can be concluded from Fig.4 that the actual trajectory is in accordance with the assumed trajectory, the trajectory is smooth and so easy to carry out; Fig.5 gives the time distribution figure of the two formation arrive at the trajectory point after time cooperation, it can be concluded that the time cooperation method can not only fulfill the time cooperation of each formation, but also can obtain the time to other trajectory point, clearly the two formation strike time profile is shown.

\section{CONCLUSION AND DISCUSSION}

The paper firstly analyze the characteristics and demand of the modern air-to-ground strike as well as the deficiency of the existing path planning method, then proposes a new path planning method focused as path planning and time cooperation. Finally, carries out a simulation based on a designed scenario, the result proves the effectiveness of the method.

\section{REFERENCES}

[1] W. Xue and H.-L. Wang, Research on Air-to-Ground Attack of UAV Based on Integrated Control.Electronics Optics and Control, vol. 17, no. 7, pp. 43-55, 2010.

[2] X.-J. Shun, Simulation of UAV Air-to-Surface attack, Northwestern Polytechnical University. Xian, China, 2003.

[3] X.-Y. Liu, X.-L. Huang and Q. Wu, Function architecture framework study on air-to-ground attack mission planning system, Journal of HarBin Institute of Technology, vol. 39, No.7, pp. 1013 1016, 2007

[4] W.Qi, "Research of aircraft route planning and evaluation algorithm” . Harbin Institute of Technology, July 2007, P7-P19.

[5] S. Karaman and E. Frazzoli, "Incremental sampling-based algorithms for optimal motion planning," in Proc. Robot. Sci. Syst, May 2010.

[6] J. Ichnowski and R. Alterovitz, "Parallel sampling-based motion planning with superlinear speedup," in Proc. IEEE/RSJ Int. Conf. Intell. Robot. Syst., Oct. 2012, pp. 1206-1212.

[7] J. J. Kuffner, "Effective sampling and distance metrics for 3D rigid body path planning," in Proc. IEEE Conf. Robot. Automat, Apr./May 2004, vol. 4, pp. 3993-3998.

[8] Ma, G., Duan, H., and Liu, S.Improved ant colony algorithm for global optimal trajectory planning of UAV under complex environment. International Journal of Computer Science and Applications, 4, 3 (2007), 57-68.

[9] Geiger, B., etal, Optimal path planning of UAVs using direct collocation with nonlinear programming.AIAA Guidance, 
Navigation, and Control Conference, Keystone, CO, Aug. 2006, AIAA Paper 2006-6199.

[10] Becker, M., Dantas, C., and Macedo, W.Obstacle avoidance procedure for mobile robots. In ABCM Symposium Series in Mechatronics (1st ed.), vol. 2, Brazilian Society of Mechanical Sciences and Engineering, Rio de Janeiro, 2006, pp. 250-257.

[11] Zhang C D, H su H T, W u X P, et a.l An alternative algebraic algorithm to transform Cartesian to geodetic coordinates. J. G. eod. , 2005, 79: 413- 420.
[12] HUANG Xiying, SHAO Wei. Modeling and Application of Threedimensional Special Data Field for Radar Detection Range ,2012 International Conference on Computer Science and Electronics Engineering. 23-25 March 2012 . (Volume:1 ) 481-483 Hangzhou. 